\title{
A NOTE ON DIRECT SUMS OF QUASINILPOTENT OPERATORS
}

\author{
L. A. FIALKOW
}

ABSTRACT. Let 2 denote the set of all qua sinilpotent operators on a fixed, separable Hilbert space $H$. D. Herrero has found necessary conditions for an operator to belong to the norm closure of 2 in $\mathscr{L}(\mathcal{H})$. We prove that each direct sum (or direct integral) of operators in $2^{-}$ satisfies these conditions; two questions of D. Herrero are thereby related to one another. We also prove that the spectrum of a direct sum of nilpotent operators may be multiply connected.

1. Introduction. Let $\mathcal{H}$ denote a complex, separable Hilbert space, and let $\mathscr{L}(\mathcal{H})$ denote the algebra of all bounded linear operators on $\mathcal{H}$. An operator $T$ in $\mathscr{L}(\mathcal{H})$ is said to be quasinilpotent if the spectrum of $T$ is the singleton $\{0\}$. Let 2 denote the set of all qua sinilpotent operators in $\mathfrak{L}(\mathcal{H})$. In [5, Theorem 1.3], Herrero proved that certain conditions are satisfied by each operator that belongs to the norm closure of 2 in $\mathfrak{L}(\mathcal{H})$; Question 1 of [5] asks whether these conditions are also sufficient for membership in $2^{-}$. Question 3 of [5] asks whether $2^{-}$is closed under infinite direct sums (in a sense to be made precise below). These questions were actually posed in the setting of Banach algebras, but will be discussed here only in the context of $\mathscr{\varrho}(\mathcal{H})$.

One purpose of this note is to relate these two questions by proving that each countable direct sum of operators in $2^{-}$does satisfy the necessary conditions of [5]. An extension of this result to direct integrals is given. We also prove that the resolvent set of a direct sum of nilpotent operators may contain countably many components. If $T$ is in $\mathscr{L}(\mathcal{H})$, let $\sigma(T)$ denote the spectrum of $T$ and let $E(T)$ denote the essential spectrum of $T$, i.e. $E(T)=\{\lambda \in \mathbf{C} \mid T-\lambda$ is not Fredholm $\}$ (see [3]). We now state for reference the necessary conditions of [5], specialized to the case of a separable Hilbert space. Each operator in $2^{-}$satisfies the following properties:

Received by the editors January 3, 1974.

AMS (MOS) subject classifications (1970). Primary 47A10, 47 A60; Secondary $47 \mathrm{~B} 99$.

Key words and phrases. Quasinilpotent operator, connected spectrum, connected essential spectrum. 
(1) $\sigma(T)$ and $E(T)$ are connected.

(2) $E(T)$ contains 0 .

(3) If $T-\lambda$ is semi-Fredholm, then $T-\lambda$ is Fredholm with index equal to zero (see [3]).

Remark. The preceding formulation of the necessary conditions is due to N. Salinas [8]. The author wishes to thank Professor Salinas for making available to the author a copy of his seminar notes and preprint of his paper on this subject.

2. Direct sums of operators in $\mathcal{2}^{-}$. Let $\left\{\mathcal{H}_{n}\right\}$ denote a sequence of separable Hilbert spaces, and let $T_{n}$ denote an operator in $\mathscr{L}\left(\mathcal{H}_{n}\right)$. If the sequence $\left\{\left\|T_{n}\right\|\right\}$ is bounded, we may define the direct sum $T=\Sigma \bigoplus T_{n}$, which is a bounded operator on the direct sum $\mathcal{H}=\Sigma \bigoplus \mathcal{H}_{n}$. If $S=\bigcup_{n} \sigma\left(T_{n}\right)$, then clearly $S \subset \sigma(T)$.

Lemma 2.1. Each nonempty closed-and-open subset of $\sigma(T)$ has nonempty intersection with $S$.

Proof. Let $X$ denote a proper, nonempty, closed-and-open subset of $\sigma(T)$. If $X$ is disjoint from $S$, there is an open set $U$ such that $\sigma(T)-$ $X \subset U$ and $X$ is disjoint from $U^{-}$. We may assume that the boundary, $B$, of $U$ consists of a finite disjoint union of rectifiable Jordan curves (see [6, pp. 417-418]). It follows from [6, Theorem p. 421] that $J=\int_{B}(T-\lambda)^{-1} d \lambda$ is a nontrivial idempotent in $\mathcal{L}(\mathcal{H})$, i.e. $J \neq 0,1_{\mathcal{H}} \cdot$ For each integer $m>0$ let $\left\{\lambda_{i, m}\right\}_{i=1}^{k}$ denote a partition of $B$ whose mesh is less than $1 / m$, and let

$$
R_{m}=\sum_{i=1}^{k_{m}-1}\left(T-\lambda_{i, m}\right)^{-1}\left(\lambda_{i+1, m}-\lambda_{i, m}\right)
$$

denote the corresponding Riemann sum. For each $\lambda$ in $B$, we have $(T-\lambda)^{-1}=$ $\Sigma \bigoplus\left(T_{n}-\lambda\right)^{-1}$, and therefore $R_{m}$ is the direct sum of Riemann sums of the form

$$
R_{m, n}=\sum_{i=1}^{k_{m}-1}\left(T_{n}-\lambda_{i, m}\right)^{-1}\left(\lambda_{i+1, m}-\lambda_{i, m}\right)
$$

For each $n$, we have $\sigma\left(T_{n}\right) \subset \sigma(T)-X \subset U$, and therefore [6, Theorem, p. 421] implies that for each $n, \lim \left\|R_{m, n}-1_{\mathcal{H}_{n}}\right\|=0$. Now, since $R_{m}=$ $\Sigma \bigoplus R_{m, n}$ and $\lim \left\|R_{m}-J\right\|=0$, it follows that $J=1_{\mathcal{H}}$, which is a contradiction. 
Corollary 2.2. If each $\sigma\left(T_{n}\right)$ is connected and $C$ is a connected subset of $S$ such that $\sigma\left(T_{n}\right) \cap C$ is nonempty for each $n$, then $\sigma(T)$ is connected.

Corollary 2.3. If each $T_{n}$ is in $2^{-}$(with respect to $\mathcal{H}_{n}$ ), then $\sigma(T)$ is connected.

Proof. Since $T_{n}$ is in $2^{-}, \sigma\left(T_{n}\right)$ is connected and contains 0 . The proof is completed by an application of the preceding corollary.

We note that the analogue of Lemma 2.1 for essential spectra is false. Indeed, if each $T_{n}$ is a finite rank projection, then $E\left(T_{n}\right)=\{0\}$ and $E(T)$ $=\{0,1\}$. Lemma 2.1 and its corollaries can be proved in the context of Banach algebras with identity, even with arbitrary (not necessarily countable) direct sums. At the end of this section we give generalizations of the above results to upper triangular matrices $w$ ith operat or entries.

Lemma 2.4. If each $T_{n}$ is quas inilpotent, then $E(T)=\sigma(T)$.

Proof. Since $E(T)$ is a nonempty subset of $\sigma(T)$ we may assume that $T$ is not quasinilpotent. It suffices to prove that if $T-\lambda$ is Fredholm, then $T-\lambda$ is invertible. If $\lambda \neq 0$, each $T_{n}-\lambda$ is invertible and, therefore, $T-\lambda$ is one-to-one and has dense range. If, in addition, $T-\lambda$ is Fredholm, then $T-\lambda$ has closed range and is thus invertible. Lemma 2.1 implies that 0 is not an isolated point of $\sigma(T)$, and since $E(T)$ is a closed set which contains each nonzero member of $\sigma(T)$, it follows that 0 belongs to $E(T)$.

Theorem 2.5. If each $T_{n}$ is in $\mathcal{2}^{-}$(with respect to $\mathcal{H}_{n}$ ), then $T$ satisfies conditions (1)-(3).

Proof. Corollary 2.3 implies that $\sigma(T)$ is connected. The hypothes is implies that $T$ is the norm limit of a sequence of operators, $\left\{S_{k}\right\}$, each of which is the direct sum of quasinilpotent operators. Corollary 2.3 and Lemma 2.4 imply that for each $k, E\left(S_{k}\right)$ is a connected set that contains 0 . Since the set of Fredholm operators on $\mathcal{H}$ is open (see [3, Corollary 1.3]), it follows that 0 is in $E(T)$. Since each $E\left(S_{k}\right)$ is connected, the upper semicontinuity of the separated parts of the essential spectrum [7, Theorem $3]$ implies that $E(T)$ is connected. To prove that condition 3 is satisfied, suppose that $T-\lambda$ is semi-Fredholm. Then clearly each $T_{n}-\lambda$ is semiFredholm, and since $T_{n}$ is in $2^{-}$, condition 3 implies that $T_{n}-\lambda$ is Fredholm with index equal to zero. Therefore the nullity and corank of $T$ $-\lambda$ are equal and the proof is complete. 
We next consider generalizations of the preceding results to upper triangular matrices with operator entries. If $T$ is of this form, then $T=$ $\left(T_{i j}\right)$, with $T_{i j}=0$ for $i>j$. Let $T_{i}=T_{i i}$ and $S=\bigcup \sigma\left(T_{i}\right)$. In [5, Theorem 1.5], Herrero proved that a finite upper triangular matrix with diagonal entries in $2^{-}$is also in $2^{-}$, while if the matrix is infinite, this is not always true. Lemma 2.1 can be extended to upper triangular operator matrices as follows.

Proposition 2.6. Each nonempty closed-and-open subset of $\sigma(T)$ has nonempty intersection with $S^{-}$.

Proof. If $X$ is a nonempty, closed-and-open subset of $\sigma(T)$ that is disjoint from $S^{-}$, we may separate $X$ from $(\sigma(T)-X) \cup S^{-}$by an open set $U$ as in the proof of Lemma 2.1. If $\lambda$ is in the boundary of $U$, then $(T-\lambda)^{-1}$ is an upper triangular operator matrix whose diagonal entries are $\left(T_{n}-\lambda\right)^{-1}$. The proof now procedes exactly like that of Lemma 2.1 ; note that an upper triangular idempotent operator matrix with all identity operators on the diagonal is equal to the identity.

Corollary 2.3 is also true for upper triangular operator matrices. Let $\pi(R)$ denote the approximate point spectrum of an operator $R$. If $T$ is an upper triangular operat or matrix such that $\sigma\left(T_{n}\right)=\pi\left(T_{n}\right)$, for each $n$, then $S \subset \sigma(T)$, and it follows that Corollary 2.3 is valid for $T$. This argument applies if each $T_{n}$ is in $2^{-}$, since condition 3 implies that $\sigma\left(T_{n}\right)=\pi\left(T_{n}\right)$ for each $n$. In general, $S$ is not contained in $\sigma(T)$ (although $\pi\left(T_{1}\right) \subset \sigma(T)$, it may happen that $\sigma\left(T_{i}\right)$ is disjoint from $\sigma(T)$ for each $\left.i>1\right)$. For this reason Corollary 2.2 cannot be extended to upper triangular operator matrices. Indeed, the simple bilateral shift, $B$, may be represented as a $2 \times 2$ upper triangular operator matrix such that each diagonal element has spectrum equal to the closed unit disk. Then $A=B \oplus 1 / 2 B$ is a $4 \times 4$ upper triangular matrix. The spectrum of $A$ consists of concentric circles of radii $1 / 2$ and 1 , while the intersection of the spectra of the four operators on the diagonal is the closed disk of radius $1 / 2$.

We conclude this section by giving an extension of the preceding results to direct integrals. We use the notation and definitions of [1]. Let $(Z, \nu)$ denote a standard measure space. For $z$ in $Z$, let $\mathcal{H}(z)=\mathcal{H}$ and $\mathcal{H}_{0}=$ $\int^{\oplus} H(z)$. For an essentially bounded family $\{T(z)\}_{z \in Z}$, with $T(z)$ in $\mathfrak{L}(\mathcal{H}(z))$, let $T=\int^{\oplus} T(z)$ denote the direct integral. Note that $\sigma(T(z))$ might not be contained in $\sigma(T)$.

Lemma 2.7. Each nonempty closed-and-open subset of $\sigma(T)$ has nonempty intersection with $\left(\bigcup_{Z} \sigma(T(z))\right)^{-}$. 
Proof. We employ the notation of Lemma 2.1 and modify its proof as follows. Fix $m>0$ and $1 \leq i \leq k_{m}$. Since $T-\lambda_{i, m}$ is invertible, Lemma 4.1 of [1] implies that there exists a subset $E_{i, m} \subset Z$ such that $\nu\left(E_{i, m}\right)=$ $0,\left(T(z)-\lambda_{i, m}\right)^{-1}$ exists for all $z \in E_{i, m}^{\prime}$, and $\left(T-\lambda_{i, m}\right)^{-1}=$ $\int_{E_{i, m}^{\prime}}^{\oplus}\left(T(z)-\lambda_{i, m}\right)^{-1}$. If $E=\bigcup_{m=1}^{\infty} \bigcup_{i=1}^{k_{m}} E_{i, m}$, then $\nu(E)=0$, and it follows that for $m>0$,

$$
R_{m}=\int_{E^{\prime}}^{\oplus} \sum_{i=1}^{k_{m}-1}\left(T(z)-\lambda_{i, m}\right)^{-1}\left(\lambda_{i+1, m}-\lambda_{i, m}\right) .
$$

Since $R_{m} \rightarrow J$, and since the decomposable operators form a von Neumann subalgebra of $\mathcal{L}\left(\mathcal{H}_{0}\right), J$ is a direct integral of the form $J=\int \oplus_{P(z)}$. It follows readily that $P(z)=1_{H(z)}$ a.e. $(\nu)$, and therefore $J=1_{H_{0}}$. Since $J$ is a nontrivial idempotent, this contradiction completes the proof.

Corollary 2.8. If each $T(z)$ is in 2, then $\sigma(T)$ is connected.

Lemma 2.9. If each $T(z)$ is quasinilpotent, then $E(T)=\sigma(T)$.

Proof. If $\lambda \neq 0$, then each $T(z)-\lambda$ is invertible, and therefore $T$ is one-to-one and has dense range. The proof proceeds like that of Lemma 2.4.

The following result is due to E. Azoff, who has kindly allowed us to reproduce it here. The proof, which is based on the "principle of measurable choice"' (see [1]), will be omitted.

Theorem 2.10 (Azoff [2]). If each $T(z)$ satisfies condition 3, then $T$ satisfies condition 3 .

Using the preceding results, the next theorem may be proven exactly like Theorem 2.5.

Theorem 2.11. If each $T(z)$ is in $2^{-}$(with respect to $\mathcal{H}(z)$ ), then $T$ satisfies conditions (1)-(3).

3. Multiply connected spectra. In this section we adapt the technique of [5, Theorem 3.7] to show that the spectrum of a direct sum of nilpotent operators may be multiply connected. Let $N_{k}$ denote the $k \times k$ nilpotent matrix of the form

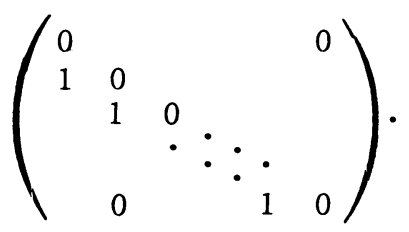


Problem 81 of [4] proves that the spectrum of $N=\Sigma_{k=2}^{\infty} \bigoplus N_{k}$ is the closed unit disk $D$.

Example 3.1. Let $C_{0}$ denote the unit circle, and for each integer $n>$ 0 let $C_{n}$ denote the circle centered at $\left(1 / 4+1 / 2^{n}, 0\right)$ with radius $1 / 2^{n+2}$. Let $D_{n}$ denote the closed disk bounded by $C_{n}$. Set $L_{0}=[7 / 8,1], L_{-1}=\{1 / 4\}$, and for $n>0$ set $L_{n}=\left[1 / 4+1 / 2^{n+1}+1 / 2^{n+3}, 1 / 4+1 / 2^{n}-1 / 2^{n+2}\right]$. Then $B=\bigcup_{n=0}^{\infty}\left(C_{n} \cup L_{n-1}\right)$ is a rectifiable curve which bounds the simply connected doma in $S=D-\left(C_{0} \cup \bigcup_{n=1}^{\infty}\left(D_{n} \cup L_{n-2}\right)\right)$. Let $f(z)=\sum_{n=1}^{\infty} a_{n} z^{n}$ be a conformal mapping of the open unit disk onto $S$. Since $B$ is a rectifiable curve, [10, Theorem 10.14, p. 293] implies that $\sum_{n=1}^{\infty}\left|a_{n}\right|<\infty$. Then Theorem 2.6 of [5] implies that $f(N)=\sum_{n=1}^{\infty} a_{n} N^{n}$ is def ined and that $\sigma(f(N))=f(\sigma(N))=S^{-}$. Therefore $\mathrm{C}-\sigma(f(N))$ has countably many components. Finally, since $f(0)=0, f(N)$ is the direct sum of nilpotent ope rators.

Let $X$ denote a compact, connected subset of the plane that contains the origin. The method of the above example can be modified to prove that if bdry $(X)$ is rectifiable, then $X$ is the spectrum of a direct sum of nilpotent operators. The following result, due to N. Salinas [9], shows that this restriction on bdry $(X)$ is unnecessary.

The orem 3.2. $X$ is the spectrum of a direct sum of nilpotent operators.

Proof. Let $N$ be a normal operator on $\mathcal{H}$ such that $\sigma(N)=X$. [5, Theorem 7] implies that there exists a sequence $\left\{N_{k}\right\}$ of nilpotent operators such that $N_{k} \rightarrow N$. Let $\mathcal{K}$ denote the direct sum of countably many copies of $\mathcal{H}$ and let $T=\Sigma_{k=1}^{\infty} \bigoplus N_{k}$. For each integer $n>0$, let $T_{n}$ be the operator on $\mathcal{K}$ of the form $T_{n}=N_{1} \oplus \cdots \oplus N_{n} \oplus S \oplus \cdots \oplus S \oplus \cdots$ Now $T_{n} \rightarrow T$, and since each $\sigma\left(T_{n}\right)=X$, it follows that $X \subset \sigma(T)$. Since the mapping $R \rightarrow R^{-1}$ is norm continuous when restricted to the set of all invertible operators on $\mathcal{K}$, it follows that $\sigma(T) \subset X$, and the proof is complete.

\section{REFERENCES}

1. E. A. Azoff, Spectrum and direct integral, Trans. Amer. Math. Soc. 197 (1974), $211-223$.

2. Private communication.

3. P. A. Fillmore, J. G. Stampfli and J. P. Williams, On the es sential numerical range, the essential spectrum, and a problem of Halmos, Acta. Sci. Math. (Szeged) (1974), 211-223.

4. P. R. Halmos, A Hilbert space problem book, Van Nostrand, Princeton, N.J., 1967. MR $34 \# 8178$.

5. D. A. Herrero, Normal limits of nilpotent operators, Indiana Univ. Math. J. (to appear). 
6. F. Riesz and B. Sz.-Nagy, Leçons d'analyse fonctionnelle, Akad. Kiadó, Budapest, 1953; English transl., Functional analysis, Ungar, New York, 1955.

MR 15, 132; 17, 175 .

7. N. Salinas, Operators with essentially disconnected spectrum, Acta. Sci. Math. (Szeged) 33 (1972), 193-205.

8. - Subnormal limits of nilpotent operators, Acta. Sci. Math. (Szeged) (to appear).

9. - Private communication.

10. A. Zygmund, Trigonometric series. Vols. I, II, 2nd rev. ed., Cambridge Univ. Press, New York, 1959. MR 21 \#698.

DEPARTMENT OF MATHEMATICS, WESTERN MICHIGAN UNIVERSITY, KALAMAZOO, MICHIGAN 49001 